\title{
Naturally selected hepatitis C virus polymorphisms confer broad neutralizing antibody resistance
}

\author{
Justin R. Bailey, ${ }^{1}$ Lisa N. Wasilewski, ${ }^{1}$ Anna E. Snider, ${ }^{1}$ Ramy El-Diwany, ${ }^{1}$ William O. Osburn, ${ }^{1}$ Zhenyong Keck, ${ }^{2}$ \\ Steven K.H. Foung, ${ }^{2}$ and Stuart C. Ray ${ }^{1,3}$ \\ 'Division of Infectious Diseases, Department of Medicine, Johns Hopkins University School of Medicine, Baltimore, Maryland, USA. ²Department of Pathology, Stanford University School of Medicine, \\ Stanford, California, USA. ${ }^{3}$ Department of Oncology, Johns Hopkins University School of Medicine, Baltimore, Maryland, USA.
}

\begin{abstract}
For hepatitis C virus (HCV) and other highly variable viruses, broadly neutralizing mAbs are an important guide for vaccine development. The development of resistance to anti-HCV mAbs is poorly understood, in part due to a lack of neutralization testing against diverse, representative panels of HCV variants. Here, we developed a neutralization panel expressing diverse, naturally occurring HCV envelopes (E1E2s) and used this panel to characterize neutralizing breadth and resistance mechanisms of 18 previously described broadly neutralizing anti-HCV human mAbs. The observed mAb resistance could not be attributed to polymorphisms in E1E2 at known mAb-binding residues. Additionally, hierarchical clustering analysis of neutralization resistance patterns revealed relationships between mAbs that were not predicted by prior epitope mapping, identifying 3 distinct neutralization clusters. Using this clustering analysis and envelope sequence data, we identified polymorphisms in E2 that confer resistance to multiple broadly neutralizing mAbs. These polymorphisms, which are not at mAb contact residues, also conferred resistance to neutralization by plasma from HCV-infected subjects. Together, our method of neutralization clustering with sequence analysis reveals that polymorphisms at noncontact residues may be a major immune evasion mechanism for HCV, facilitating viral persistence and presenting a challenge for HCV vaccine development.
\end{abstract}

\section{Introduction}

Despite recent advances in hepatitis C virus (HCV) treatment, a vaccine against the virus is still urgently needed (1-3). Vaccine design is challenging due to extensive world-wide genetic diversity of the virus and rapid viral evolution in infected individuals (4-7).

Most HCV-infected individuals develop neutralizing antibodies (nAbs) against the virus, but viral evolution at highly variable loci in HCV E2 (envelope) can lead to escape from isolate-specific nAbs (8-11). However, isolation of human mAbs capable of neutralizing multiple diverse $\mathrm{HCV}$ isolates has shown that nAbs may also target more conserved regions of the E1 and E2 proteins (12-25). Discovery of these broadly neutralizing $\mathrm{mAbs}$ has raised hope that a vaccine inducing similar nAbs could prevent HCV infection.

Due to limited availability of diverse strains of replication competent $\mathrm{HCV}(\mathrm{HCVcc})$ and previously limited availability of diverse E1E2 clones for use in HCV pseudoparticles (HCVpp), the breadth of neutralization of anti-HCV mAbs has generally been measured against small panels of HCV isolates (12, 16, 26-29). In addition, epitopes of neutralizing mAbs have generally been mapped by alanine scanning mutagenesis and binding assays. While these studies provide useful information on key mAb-binding residues,

\section{Related Commentary: p. 97}

Conflict of interest: The authors have declared that no conflict of interest exists Submitted: August 29, 2014; Accepted: October 16, 2014

Reference information: J Clin Invest. 2015;125(1):437-447. doi:10.1172/JCI78794. they do not measure the neutralization resistance conferred by the vast array of HCV envelope polymorphisms that occur in nature.

Therefore, we developed a novel panel neutralization method that uses the natural variation of HCVE1E2, allowing measurement of neutralizing breadth, clustering of mAbs with similar resistance profiles, and identification of neutralization resistance polymorphisms anywhere in E1E2. Hundreds of unique E1E2 clones were isolated from individuals infected with genotype $1 \mathrm{HCV}$, and, from this library, 19 genotype 1a and 1b HCV E1E2 clones were selected to maximize sequence diversity among clones (30). The panel of 19 clones contains $94 \%$ of amino acid polymorphisms present at greater than $5 \%$ frequency in a reference panel of 643 genotype 1 HCV isolates from GenBank (31). These clones were used to produce a panel of HCVpp, which were tested for neutralization by 18 previously described broadly neutralizing anti-HCV human mAbs, including some of the most potent and broadly neutralizing mAbs described to date (refs. 12, 16-20, and Supplemental Table 1; supplemental material available online with this article; doi:10.1172/ JCI78794DS1). mAbs were grouped using hierarchical clustering analysis of pairwise neutralization resistance profile correlations and, using novel methods, E1E2 sequences were analyzed to identify polymorphisms associated with broad $\mathrm{nAb}$ resistance.

\section{Results}

Each neutralizing $m A b$ produces a neutralization profile across the HCVpp panel. Eighteen mAbs were each tested for neutralization of each of 19 clonal genotype $1 \mathrm{HCVpp}$, the largest genotype 1 panel against which these mAbs have been tested. Neutralization 
by representative $\mathrm{mAbs}$ is shown in Figure 1A, with neutralization results for all $\mathrm{mAbs}$ shown in Supplemental Figure 1. mAbs HC84.26 and AR4A showed the greatest neutralizing breadth, reducing by at least $50 \%$ the infectivity of 17 of 19 HCVpp and 18 of 19 HCVpp, respectively, at $10 \mu \mathrm{g} / \mathrm{ml} \mathrm{mAb}$. (Supplemental Figure 1). Notably, sensitivity to each mAb varied across the HCVpp panel, with some E1E2 clones highly sensitive (relative infection $<0.1$ ), some moderately resistant (relative infection 0.1-0.5), and some highly resistant (relative infection $>0.5$ ) to each $\mathrm{mAb}$. For $\mathrm{mAb}$ HC84.26, neutralization sensitivity across the panel varied by more than 1,000-fold. To validate accuracy, $\mathrm{IC}_{50} \mathrm{~s}$ of 6 of the mAbs against full-length HCVcc bearing H77 E1E2 (32) were compared with the relative infection of H77 HCVpp measured using the same mAbs. The correlation between $\mathrm{HCVcc} \mathrm{IC}_{50}$ and HCVpp relative infection was significant $(r=0.93, P<0.02$, Supplemental Figure 2). In addition, full $\mathrm{mAb}$ dilution curves were performed and $\mathrm{IC}_{50} \mathrm{~s}$ were calculated for $24 \mathrm{HCVpp} / \mathrm{mAb}$ combinations. The $\mathrm{IC}_{50} \mathrm{~s}$ correlated significantly with the relative infection measured for the same mAb/HCVpp combinations $(r=0.91$, $P<0.0001$, Supplemental Figure 3).

Ranking of resistance of library HCVpp reveals relationships between $m A b s$. To further validate the unique pattern of HCVpp panel resistance to each $\mathrm{mAb}$, rankings of HCVpp neutralization resistance to 2 closely related mAbs targeting the same linear epitope (HC33.4 and HC33.8) were compared and found to have extremely high correlation $(r=0.94, P<0.0001$, Figure 1B). Rankings of resistance to 2 unrelated mAbs (HC33.4 and HC84.22) were also compared and, as predicted, showed no correlation ( $r=0.07, P=0.75$, Figure $1 C)$. Surprisingly, a highly significant correlation was observed between the resistance profiles of mAbs HC84.22 and AR3C $(r=0.84, P<0.0001$, Figure 1D). Prior alanine scanning mutagenesis suggested that these mAbs bind to distinct epitopes (Supplemental Table 1 and refs. 12, 33). However, the recent publication by Kong and colleagues of the crystal structure of AR3C in complex with E2 core and crystal structures of $2 \mathrm{mAbs}$ related to HC84.22, HC84.1 and HC84.27, with their peptide epitopes, suggest that AR3C and HC84.22 likely bind to overlapping E2 epitopes, supporting the correlation in resistance profiles observed here $(21,34)$.

Pairwise correlations among all $\mathrm{mAb}$ resistance profiles were calculated (Figure 2). Invariably, mAbs predicted to bind overlapping epitopes based on prior alanine scanning clustered together in this analysis (i.e., HC84.22 and HC84.26; AR3A, AR3B, AR3C, and AR3D; HC33.4 and HC33.8; AR4A and AR5A), but, surprisingly, many mAbs thought to have distinct epitopes based on prior mapping (i.e., HC84.22 and AR3C; HC33.4 and AR4A) also showed significant correlation in their neutralization resistance profiles. This suggests that polymorphisms, possibly outside of known binding epitopes, may confer resistance to multiple mAbs or that multiple epitopes may be genetically linked.

Hierarchical clustering analysis using pairwise correlations of neutralization resistance profiles placed all $\mathrm{mAbs}$, except nonneutralizing $\mathrm{mAb} \mathrm{CBH}-4 \mathrm{~B}$, into 3 strongly supported neutralization clusters (Figure 2). The $8 \mathrm{mAbs}$ falling in neutralization cluster 1 (NC1), including $\mathrm{CBH}-2, \mathrm{HC} 84.26, \mathrm{CBH}-5, \mathrm{HC} 84.22$, AR3A, AR3B, AR3C, and AR3D, were chosen for further analysis.
Sequence analysis reveals polymorphisms associated with broad $m A b$ resistance. A method was developed to identify polymorphisms in E1E2 associated with $\mathrm{mAb}$ resistance. A representative analysis for mAb HC84.22 is shown in Figure 3A, and the analyses for the remaining NC1 mAbs are shown in Supplemental Figure 4. The amino acid sequence of each of the 14 E1E2 clones most resistant to each $\mathrm{mAb}$ was compared with the sequences of the 5 most sensitive E1E2 clones to identify polymorphisms present in any resistant clone but absent in all sensitive clones. Sites with substitutions in at least 2 resistant clones were included in a summary panel for each $\mathrm{mAb}$. The results for all mAbs were compiled, as shown in Figure 3B, to identify polymorphisms associated with resistance to multiple NC1 neutralizing mAbs.

Interestingly, the majority of candidate $\mathrm{NC1} \mathrm{mAb}$ resistance polymorphisms fell among positions 416-560, which span the front layer, CD81-binding loop, and the central $\beta$-sheet of E2. Polymorphisms in resistant clones relative to sensitive clones were 4-fold more likely in this region than in the remainder of the E1E2 protein $(P<0.0001)$ (Figure 3B). As expected, some of the resistance-associated polymorphisms were at positions previously identified as binding residues for NC1 mAbs (Figure 3C). For example, D431 and F442 were identified by alanine scanning as binding residues for $\mathrm{CBH}-2$ (19) and HC84 mAbs (12), respectively, and polymorphisms at those sites were also associated with resistance to those mAbs in this analysis. In addition, polymorphisms at 4 sites identified recently as contact residues for $\mathrm{mAb}$ AR3C in the E2 core/AR3C crystal structure (residues 433, 438, 442 , and 446; ref. 34 ) were also associated with resistance to mAb $\mathrm{AR} 3 \mathrm{C}$ in this analysis.

Notably, most resistance-associated polymorphisms were not at previously identified NC1 mAb-binding residues, and many neutralization-resistant E1E2 clones did not have polymorphisms at any previously identified $\mathrm{mAb}$-binding residues (Figure 3C and Supplemental Figure 4). Eleven resistance-associated polymorphisms, including D431E and F442I, were selected for further analysis.

Polymorphisms at residues 431, 442, and 560 each confer relative resistance to multiple broadly neutralizing mAbs. The 11 mutations of interest were introduced individually or in combination by sitedirected mutagenesis into an E1E2 clone, 1b09, which was sensitive to neutralization by each of the NC1 mAbs (Figure 4). Surprisingly, introduction of glutamic acid at D431, a known binding residue only for $\mathrm{CBH}-2$, conferred relative resistance to 6 of $7 \mathrm{NC} 1 \mathrm{mAbs}$, namely $\mathrm{CBH}-2, \mathrm{CBH}-5$, AR3A, AR3B, AR3C, and HC84.22. F560Y also conferred a small but significant increase in resistance to 6 of $7 \mathrm{mAbs}$. Introduction of F442I, individually or in combination with other mutations, conferred resistance to HC84.22 and HC84.26. Most of the remaining mutations tested, including N430D, L433I, L438I, $\mathrm{K} 446 \mathrm{E}$, and A475T, conferred small but statistically significant increases in resistance to mAbs HC84.22 and/or HC84.26. These same 11 mutations were also introduced into a second neutralization-sensitive E1E2 clone (1a53) with similar results (Supplemental Figure 5). When D431E and F442I were back mutated in resistant E1E2 clones, in which they occurred naturally, the predicted loss of $\mathrm{mAb}$ resistance was also observed. (Supplemental Figure 6).

Of note, while introduction of D431E into clone $1 \mathrm{~b} 09$ by sitedirected mutagenesis conferred relative resistance to 6 of $7 \mathrm{NC} 1$ 

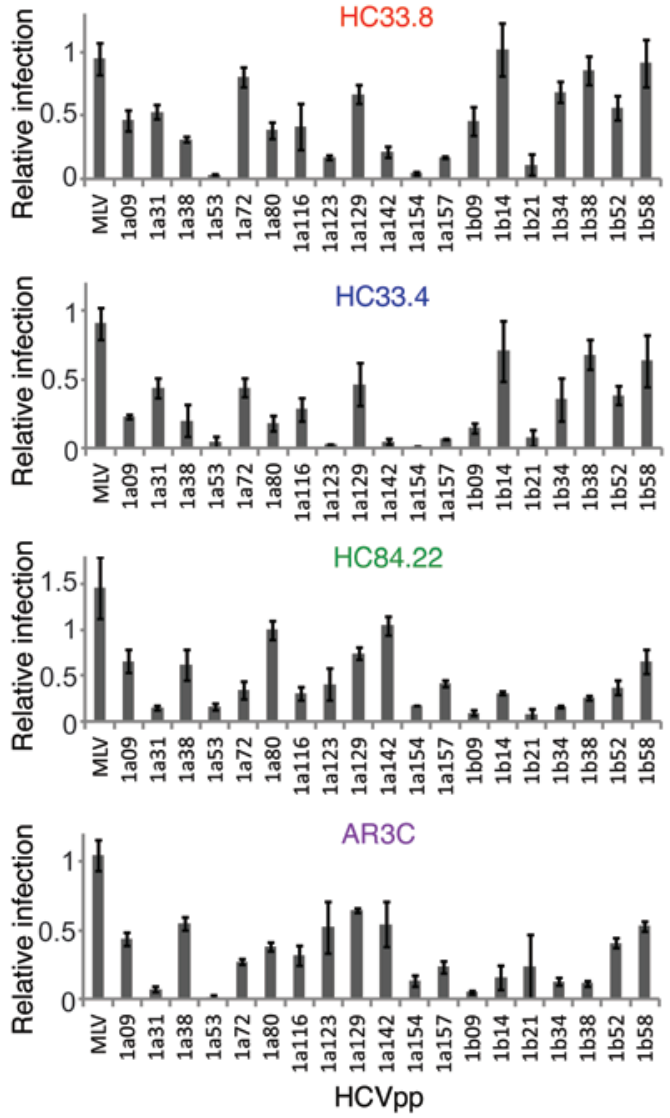

B

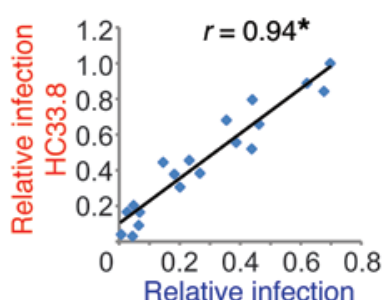

$\mathrm{HC} 33.4$

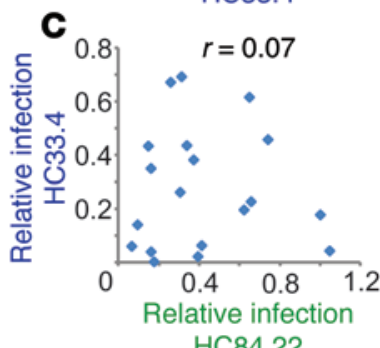

D

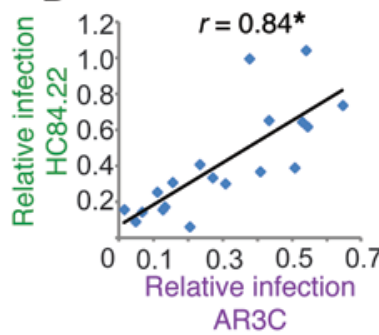

Figure 1. Ranking of library HCVpp resistance reveals relationships among mAbs. (A) Each bar represents neutralization of a unique HCVpp by the indicated $\mathrm{mAb}$. Representative mAbs are shown here, and neutralization results for all 18 mAbs are shown in Supplemental Figure 1. Relative infection is infection in the presence of $10 \mu \mathrm{g} / \mathrm{ml}$ neutralizing $\mathrm{mAb}$ relative to infection in the presence of nonspecific lgG. Error bars indicate SDs between duplicate wells. Pseudoparticles with murine leukemia virus (MLV) envelope serve as a negative control for neutralization. (B-D) Each blue diamond indicates neutralization of an individual pseudoparticle by one antibody on the $x$ axis and a second antibody on the $y$ axis. Spearman correlations ( $r$ ) are indicated for each mAb pair. ${ }^{*} P<0.0001$.

(B) Comparison of HCVpp resistance to $\mathrm{HC33.4}$ and $\mathrm{HC} 33.8,2$ closely related mAbs targeting the same linear epitope. (C) Comparison of HCVpp resistance to 2 unrelated mAbs, HC33.4 and HC84.22. (D) Comparison of HCVpp resistance to AR3C and HC84.22, 2 mAbs with distinct epitopes based on alanine scanning but overlapping epitopes based on crystal structures.

none of these polymorphisms confer high level resistance to all NC1 mAbs. Therefore, we further investigated 2 NC1-resistant E1E2 clones, 1a129 and 1a142. Clone 1a129 was resistant despite lacking F442I, D431E, and F560Y (Supplemental Figure 8). Clone 1a142 carries the F442I polymorphism, explaining its resistance to HC84.22 and HC84.26, but it also showed significant resistance to the other

mAbs, some E1E2s with naturally occurring E431 were sensitive to most NC1 mAbs (Supplemental Figures 7 and 8), suggesting that E1E2 context, the combined effect of all polymorphisms in individual E1E2 clones, can influence the resistance phenotype of D431E. In addition, although F442 is an AR3C contact residue in the AR3C/E2 crystal structure, introduction of F442I into clone $1 \mathrm{~b} 09$ did not confer detectable resistance to AR3C, and back mutation of isoleucine to phenylalanine at 442 in an E1E2 clone in which $\mathrm{I} 442$ occurred naturally did not increase sensitivity to AR3C (Figure 4, Supplemental Figure 6, and ref. 34). However, introduction of F442I or F442L into a different sensitive E1E2 clone, 1a53, did confer AR3C resistance (Supplemental Figure 5). This suggests that resistance conferred against some mAbs by F442I is also dependent on E1E2 context.

As shown in Supplemental Figure 9, introduction of D431E, F442I, or F560Y into clone 1b09 reduces HCVpp infectivity, with F442I reducing entry more than 10 -fold. Interestingly, introduction of additional polymorphisms L438I, A475T, and P453S along with F442I partially restores infectivity. Most importantly, infectivity of some HCVpp produced with E1E2 clones containing naturally occurring D431E, F442I, or F560Y polymorphisms was greater than the median infectivity of the HCVpp panel (Supplemental Figure 9B), suggesting that compensatory mutations can reverse the fitness cost of these polymorphisms.

Polymorphisms in the central $\beta$-sheet confer broad resistance. While D431E, F442I, and F560Y polymorphisms may partially explain the correlation in resistance profiles among NC1 mAbs,
NC1 mAbs (Figure 1 and Supplemental Figure 1), which cannot be attributed to F442I. To identify polymorphisms responsible for this resistance, the sequence encoding the E2 front layer, $\beta$-sheet, or front layer/CD81-binding loop/ $\beta$-sheet from resistant E1E2 clones 1a129 and 1a142 was transferred into sensitive E1E2 clone 1b09. These E1E2 chimeras are referred to herein as 1b09/1a129-F or 1b09/1a142-F, 1b09/1a129- $\beta$ or 1b09/1a142- $\beta$, and $1 \mathrm{~b} 09 / 1 \mathrm{a} 129-\mathrm{FC} \beta$ or $1 \mathrm{~b} 09 / 1 \mathrm{a} 142-\mathrm{FC} \beta$, respectively. E1E2 chimeras 1b09/1a129-F and 1b09/1a142-FC $\beta$ were poorly functional in HCVpp so were not studied further. The remaining 4 chimeras were functional.

As shown in Figure 5, resistance to HC84.22, AR3A, and $\mathrm{CBH}-2$ was conferred by transfer of the sequence encoding amino acids 526-569 ( $\beta$-sheet) from resistant clone 1a129 into sensitive E1E2 clone $1 \mathrm{~b} 09$ (15- to 55 -fold increase in resistance). This was also true when the same region ( $\beta$-sheet) was transferred from resistant clone 1a142 into sensitive E1E2 clone 1b09 (4- to 8-fold increase in resistance, Figure 6). Figure 6 also shows that amino acids $412-444$ (front layer) from clone 1a142, including F442I, confer a 2- to 31-fold increase in clone 1 b09 resistance to $\mathrm{HC} 84.22$, $\mathrm{CBH}-2$, and $\mathrm{HC} 84.26$, while the F442I mutation alone confers resistance to HC84.22 and HC84.26 but not to $\mathrm{CBH}-2$. Together, these results suggest that mutation at the F442 site is critical for high level HC84.26 resistance and adequate but not necessary for HC84.22 resistance. Polymorphisms in clones $1 \mathrm{a} 129$ and $1 \mathrm{a} 142$ between amino acids 526 and 569 ( $\beta$-sheet) confer resistance to $\mathrm{HC} 84.22$, AR3A, and $\mathrm{CBH}-2$. 


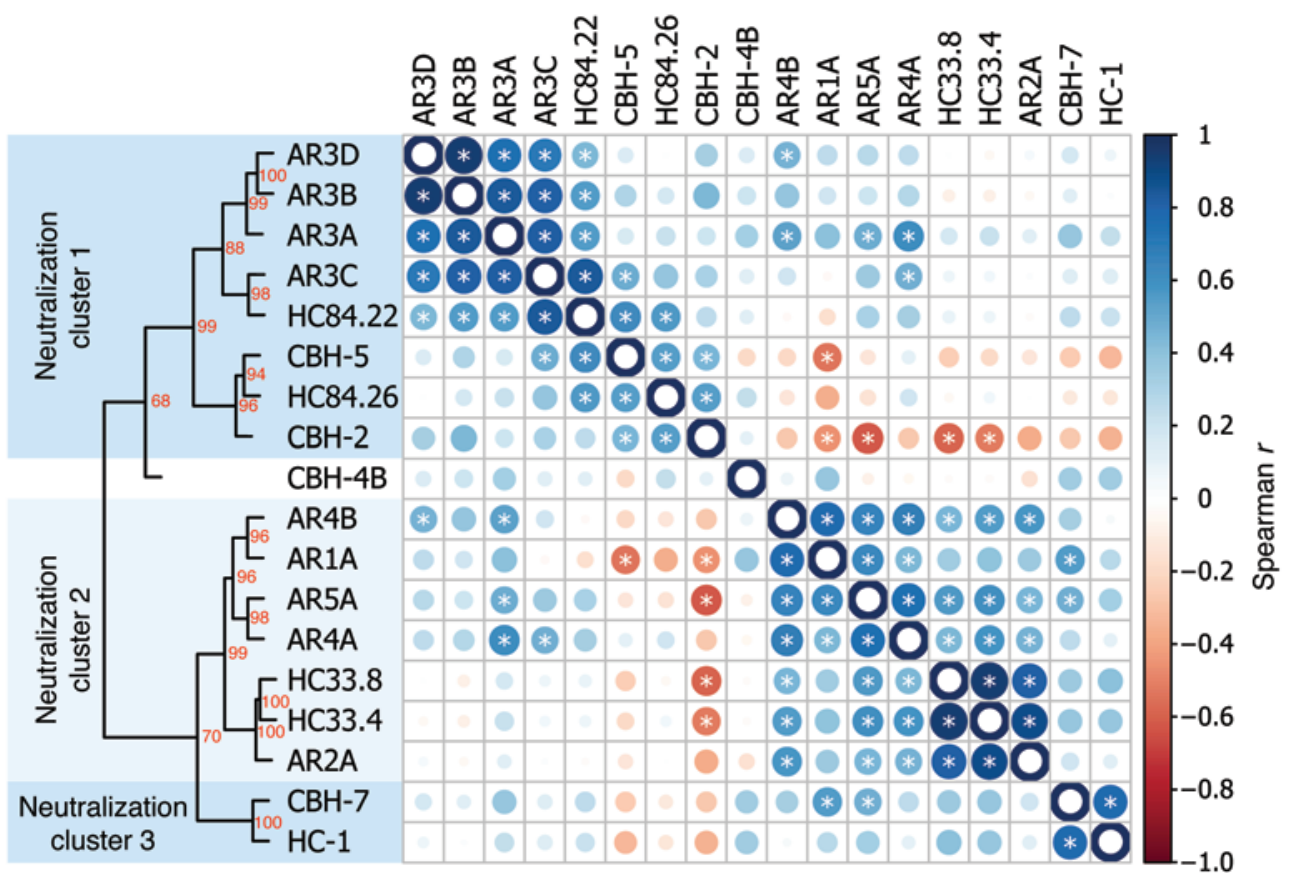

Figure 2. All neutralizing mAbs segregate into 3 neutralization clusters. A heat map was generated using pairwise correlations among all $\mathrm{mAb}$ resistance profiles. Circles at each intersection are scaled by the magnitude of the Spearman correlation $(r)$ between neutralization profiles of the indicated mAbs, with darker blue indicating higher $r$ values. Open circles indicate identity. ${ }^{*} P<0.05$. Hierarchical clustering analysis using pairwise correlations of neutralization resistance patterns is depicted as a tree. Numbers at tree nodes are approximately unbiased (AU) test values(42), which indicate strength of support for a particular cluster.
Sequence analysis of amino acids 526-569 in resistant E1E2 clones 1a129 and 1a142 relative to sensitive clone $1 \mathrm{~b} 09$ revealed 7 in-common variant sites in the resistant clones (Figure 7A). Each of these 7 polymorphisms in the 1b09/1a129- $\beta$ chimera were back mutated individually or in combination to match clone $1 \mathrm{~b} 09$ to identify the polymorphisms responsible for the broad resistance of the $1 \mathrm{a} 129$ and $1 \mathrm{a} 142$ clones. I538V, Q546L, and T563V in the central $\beta$-sheet were critical for maintaining the $\mathrm{mAb}$ resistance of the 1b09/1a129- $\beta$ chimera (Figure 7B). Back mutation at any of these 3 positions resulted in a 2 - to 3 -fold increase in sensitivity to $\mathrm{mAbs}$ CBH-2, CBH-5, AR3A, and HC84.22 (Figure 7C). Notably, the effect of these mutations was additive, as back mutation of V538 to I, L546 to Q, and V563 to T together resulted in 7- to 11-fold increase in sensitivity. As shown in Figure 8A, introduction of I538V/Q546L/T563V into sensitive clone 1b09 conferred relative resistance to $6 \mathrm{NC1} \mathrm{mAbs}$ (median 8.5-fold increase in resistance), and back mutation of the 3 polymorphisms in naturally resistant clone 1a129 conferred sensitivity to all NC1 mAbs (median 5-fold decrease in resistance). Introduction of $1538 \mathrm{~V} / \mathrm{Q} 546 \mathrm{~L} / \mathrm{T} 563 \mathrm{~V}$ into sensitive clone $1 \mathrm{b09}$ reduced binding of $\mathrm{NC} 1 \mathrm{mAbs} \mathrm{CBH}-2$ and $\mathrm{AR} 3 \mathrm{C}$, while back mutation of the 3 polymorphisms in naturally resistant clone $1 \mathrm{a} 129$ increased $\mathrm{mAb}$ binding (Figure $8 \mathrm{~B}$ ). Together, these data suggest that I538V/Q546L/T563V polymorphisms confer resistance to $\mathrm{NC1} \mathrm{mAbs}$ by reducing $\mathrm{mAb}$ binding.

Residues 538, 546, and 563 fall in the central $\beta$-sheet of E2, distant from the binding site of AR3C (ref. 34 and Figure 8C). None of these 3 positions are contact residues for AR3C or known binding residues for any other NC1 mAb. Introduction of I538V/ Q546L/T563V reduced entry of the 1b09 HCVpp, suggesting a potential fitness cost (Supplemental Figure 10). However, entry of HCVpp produced with E1E2 clones carrying naturally occurring I538V/Q546L/T563V, including resistant clone 1a129, was not reduced, suggesting that compensatory mutations can reverse the fitness cost of these polymorphisms. Interestingly, I538V/Q546L/
T563V was present in the majority of the genotype 1a E1E2 clones in the panel, including some clones, like 1a154 (H77), that are NC1 mAb sensitive (Supplemental Figure 11). This suggests that, as with D431E and F442I, the nAb resistance conferred by I538V/ Q546L/T563V can be E1E2 context dependent.

I538V/Q546L/T563V polymorphisms confer resistance to plasma neutralization. We also measured the effect of $\mathrm{I} 538 \mathrm{~V} /$ Q546L/T563V on neutralization by plasma from HCV-infected subjects (Figure 9). Strikingly, introduction of I538V/Q546L/ T563V into sensitive E1E2 clone 1b09 conferred resistance to neutralization by each of 18 plasma samples tested (median relative infection 0.33 vs. 0.65 after I538V/Q546L/T563V, $P=1 \mathrm{E}-4$ ). Back mutation of these polymorphisms in a naturally neutralizationresistant clone, 1a129, led to increased sensitivity to neutralization by all plasma samples tested (median relative infection 0.76 vs. 0.09 after V538I/L546Q/V563T, $P=8 \mathrm{E}-6)$. Notably, this effect on neutralization sensitivity was also observed with plasma from the source subject of resistant E1E2 clone 1a129. Together, these results suggest that this combination of polymorphisms likely confers resistance to neutralization in vivo.

\section{Discussion}

Using analysis of neutralization resistance profiles, we identified neutralization-resistant E1E2 clones and $\mathrm{mAb}$ neutralization clusters that were not predicted by prior epitope mapping, and we characterized novel polymorphisms conferring relative resistance to up to $6 \mathrm{mAbs}$ in one of these neutralization clusters. Resistance polymorphisms I538V/Q546L/T563V are not at known contact or binding residues for any of the mAbs to which they confer resistance, and they fall within the central $\beta$-sheet of E2 rather than the front layer/CD81-binding loop that is the likely binding site for NC1 mAbs. However, these polymorphisms reduce mAb binding to E1E2, suggesting that they may induce structural changes in E2 that reduce $\mathrm{mAb}$ binding and neutralization. 
A
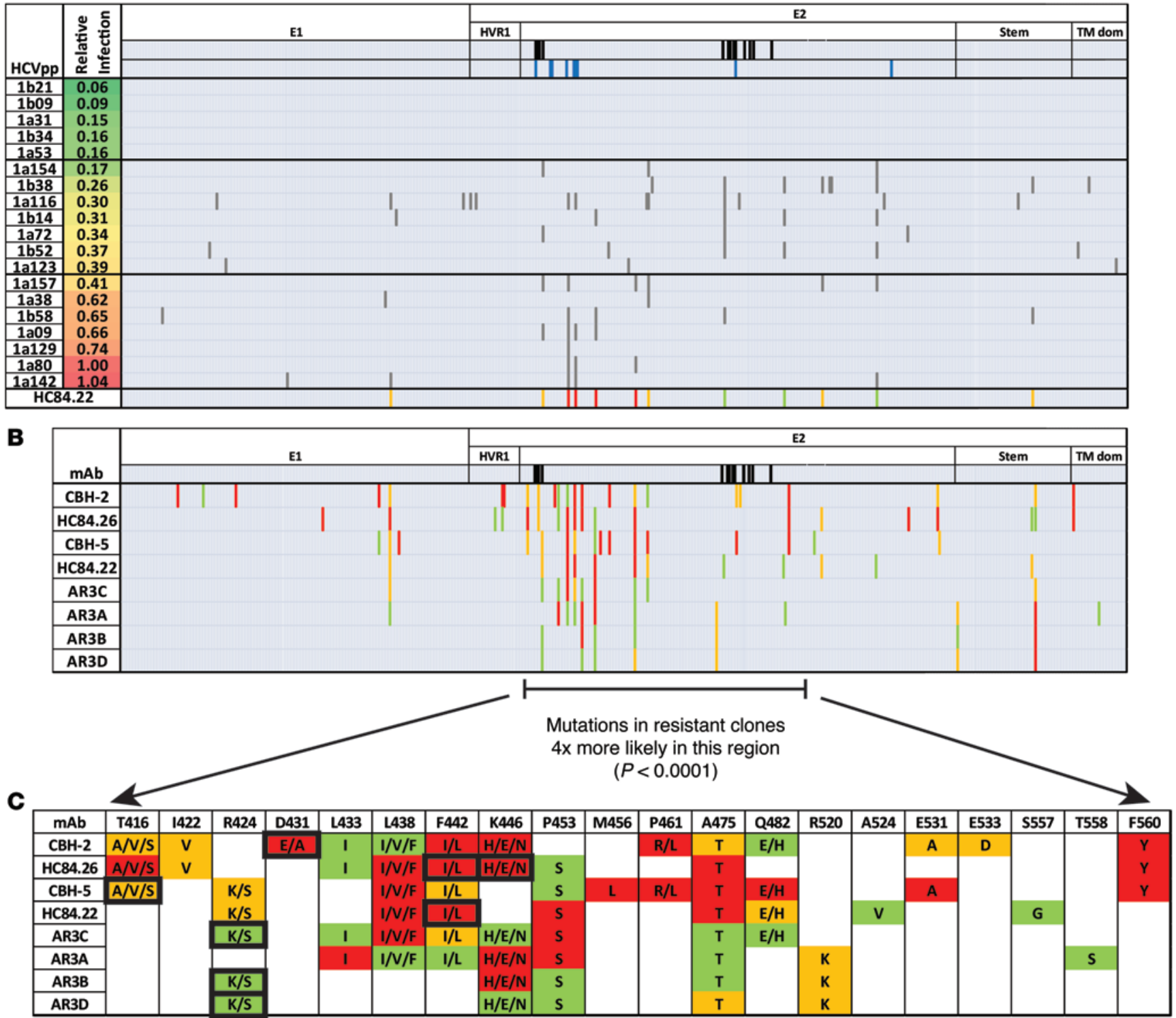

Figure 3. Sequence analysis reveals polymorphisms associated with neutralization resistance. (A) Representative E1E2 sequence analysis to identify mAb HC84.22 resistance-associated polymorphisms. Similar analyses for the remaining NC1 mAbs are shown in Supplemental Figure 4. E1E2 clones are ranked by increasing resistance to HC84.22. Clones are grouped into the 5 most sensitive, 7 with intermediate resistance, and 7 with greatest resistance, separated by horizontal black lines. Gray vertical bars indicate positions with a substitution in any resistant E1E2 clone but in none of the 5 most sensitive E1E2 clones. Black vertical bars indicate CD81-binding sites in E2 determined by alanine scanning. Blue vertical bars indicate critical binding residues for HC84.22 determined by alanine scanning. Sites with substitutions in at least 2 resistant clones but in no sensitive clones are included in the summary panel in the bottom row. (A-C) Sites marked with red are predominantly polymorphic in the 7 most resistant clones. Orange indicates sites that are polymorphic in an equal number of highly resistant and intermediate resistant clones. Green indicates sites that are predominantly polymorphic in the 7 clones with intermediate resistance. (B) Compiled results for all NC1 mAbs. (C) Resistance-associated polymorphisms, with the more commonly observed polymorphism listed first. The amino acid found at each position in the 5 most neutralization-sensitive E1E2 clones is indicated in the top row. Black boxes indicate positions at which results from this analysis are concordant with alanine scanning mutagenesis results with the same mAb.

Based on prior epitope mapping, the mAbs analyzed in this study could be divided into up to 10 distinct antigenic groups (antigenic domain A-D, antigenic region 1-5, epitope "1"). However, we show for the first time that all of these mAbs, except nonneutralizing $\mathrm{mAb} \mathrm{CBH}-4 \mathrm{~B}$, segregate into only 3 distinct neutralization clusters. This suggests that resistance polymorphisms outside of known epitopes may confer resistance to many antiHCV human mAbs, including some of the most broadly neutraliz- ing mAbs described to date. The identification of clusters of mAbs with highly correlated resistance profiles could have significant implications for HCV treatment with mAb combinations or vaccination designed to induce mAbs against multiple epitopes. This method could be used to identify combinations of mAbs with complementary resistance profiles (i.e., $\mathrm{NC} 1$ and NC2 mAbs) that may be more likely to provide additive protection when used together. A recent report by Carlsen et al. supports the potential utility of 

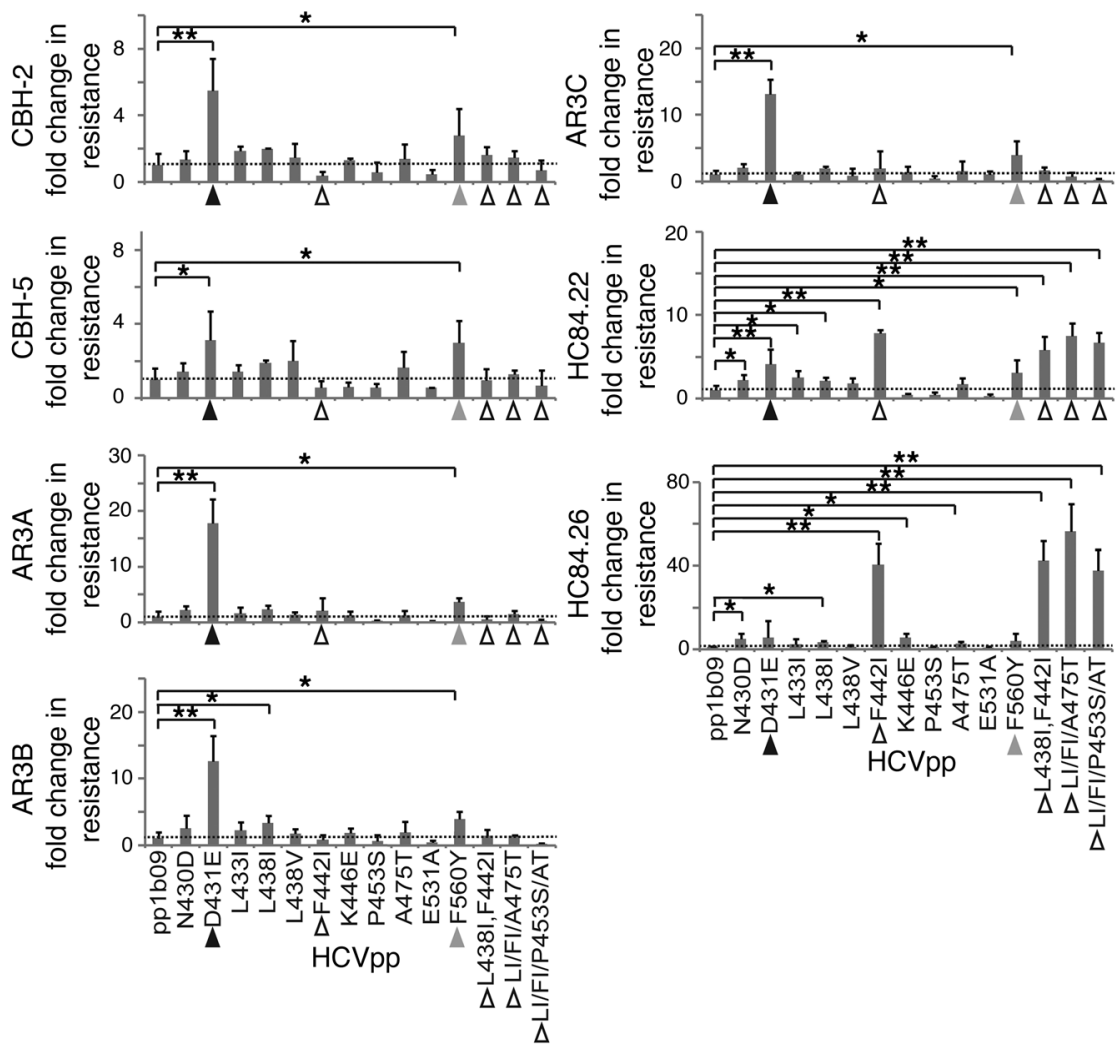

neutralization clustering, as it showed synergistic neutralization by a combination of mAbs, HC84.26 and AR4A, which we have identified as NC1 and NC2 mAbs, respectively, in this study (35).

Increasing evidence suggests that mutations outside of known binding epitopes may confer resistance to anti-HCV mAbs. The Carlsen study found no correlation between variation in mapped $\mathrm{mAb}$ epitopes and $\mathrm{mAb}$ resistance (35). In addition, studies by Keck et al. (14) and Fofana et al. (36) described mutations arising in single HCV-infected subjects that fell outside of known $\mathrm{mAb}$-binding epitopes and conferred relative resistance to multiple anti-HCV mAbs. We extend those findings by showing that
Figure 4. D431E, F442I, and F560Y mutations each confer resistance to multiple broadly neutralizing mAbs. The dashed lines indicate relative infection of wild-type 1 bog HCVpp in the presence of the indicated $\mathrm{mAb}$, adjusted to 1. Each bar indicates the fold change in HCVpp neutralization resistance relative to wild-type $1 \mathrm{~b} 09$ after the indicated mutation(s) were introduced. Values are the means of 2 to 6 independent experiments performed in duplicate, and error bars indicate SD. ${ }^{*} P<0.05,{ }^{* *} P<0.005$ by $t$ test. Black triangles indicate HCVpp with D431E mutations. White triangles indicate HCVpp with F442I mutations. Gray triangles indicated HCVpp with F560Y mutations. pp1b09, HCVpp with wildtype E1E2 1b09.

broad resistance polymorphisms at noncontact residues are a common mechanism of $\mathrm{nAb}$ resistance, as they were repeatedly observed in a representative panel of E1E2 clones generated from $19 \mathrm{HCV}$-infected subjects. We also show that these resistance polymorphisms can have additive effects and may have phenotypes that are E1E2 context dependent.

These findings are also relevant for $\mathrm{HCV}$ persistence in vivo. The I538V/Q546L/T563V polymorphisms confer resistance to neutralization by plasma from HCV-infected subjects, suggesting that these polymorphisms may contribute to $\mathrm{HCV}$ persistence during chronic infection. Importantly, identification of these resistance polymorphisms in multiple circulating E1E2 clones from multiple subjects also suggests that any fitness cost of these polymorphisms can be adequately reversed by compensatory mutations elsewhere in the virus.

Multiple resistance-associated polymorphisms, including 4 at sites identified as contact residues for AR3C in the recently published crystal structure of E2 core/AR3C (34), did not confer detectable resistance when introduced into sensitive clone $1 \mathrm{~b} 09$. This may be because most of the tested mutations are biochem-
A

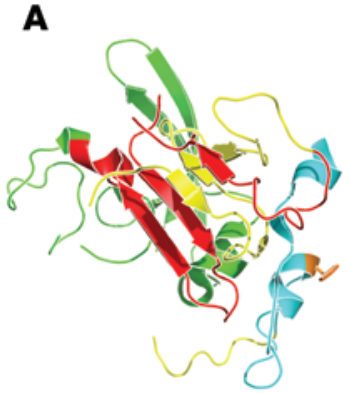

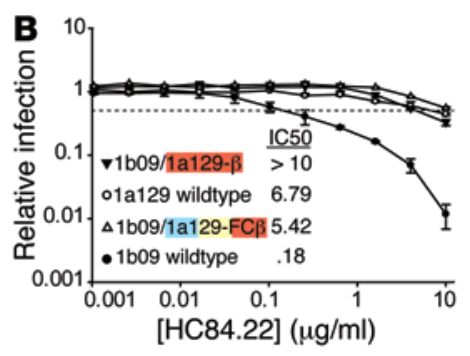

$\left[\begin{array}{ccc}0.01 & 0.1 & 1 \\ H C 84.22\end{array}\right](\mu \mathrm{g} / \mathrm{ml})$
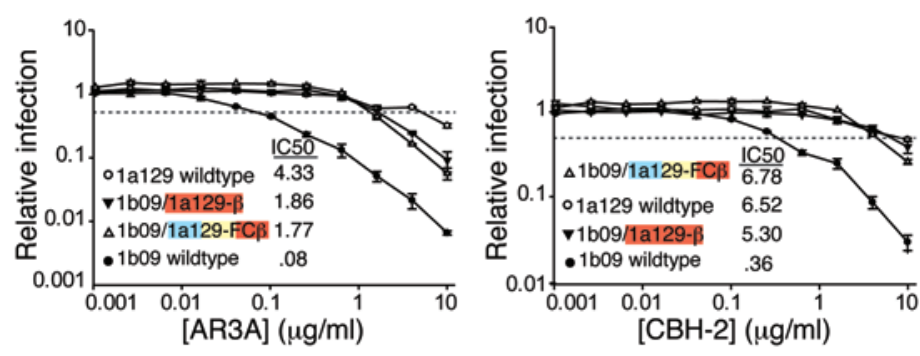

Figure 5. Polymorphisms between amino acids 526 and 569 ( $\beta$-sheet) of resistant E1E2 clone 1a129 confer resistance to multiple broadly neutralizing mAbs. (A) Crystal structure of H77 E2 core, published by Kong and colleagues (34), from the Protein Data Bank, accession 4MWF, with colors modified. E2 core front layer (amino acids $412-444$ ) is cyan, $\beta 2-\beta 5 /$ partial CD81-binding loop (amino acids $445-525$ ) is yellow, and partial CD81-binding loop/ $\beta 6$ - $\beta 8$ (amino acids 526-569) is red. Remaining structure is green. Residue 442 is marked in orange. (B) Neutralization by the indicated mAb of HCVpp with E1E2 chimeras between sensitive clone $1 \mathrm{b09}$ and neutralization-resistant E1E2 clone 1a129. Chimeras with $\beta 6-\beta 8$ (1a129- $\beta$, amino acids 526-569) from resistant clone 1 a129 are marked in red. Chimeras with the front layer, CD81-binding loop, and the central $\beta$-sheet (1a129-FC $\beta$, amino acids 412-569) from resistant clone 1 1a129 are marked with cyan, yellow, and red. Dashed lines indicate 50\% neutralization. Error bars indicate SD between duplicate wells. 

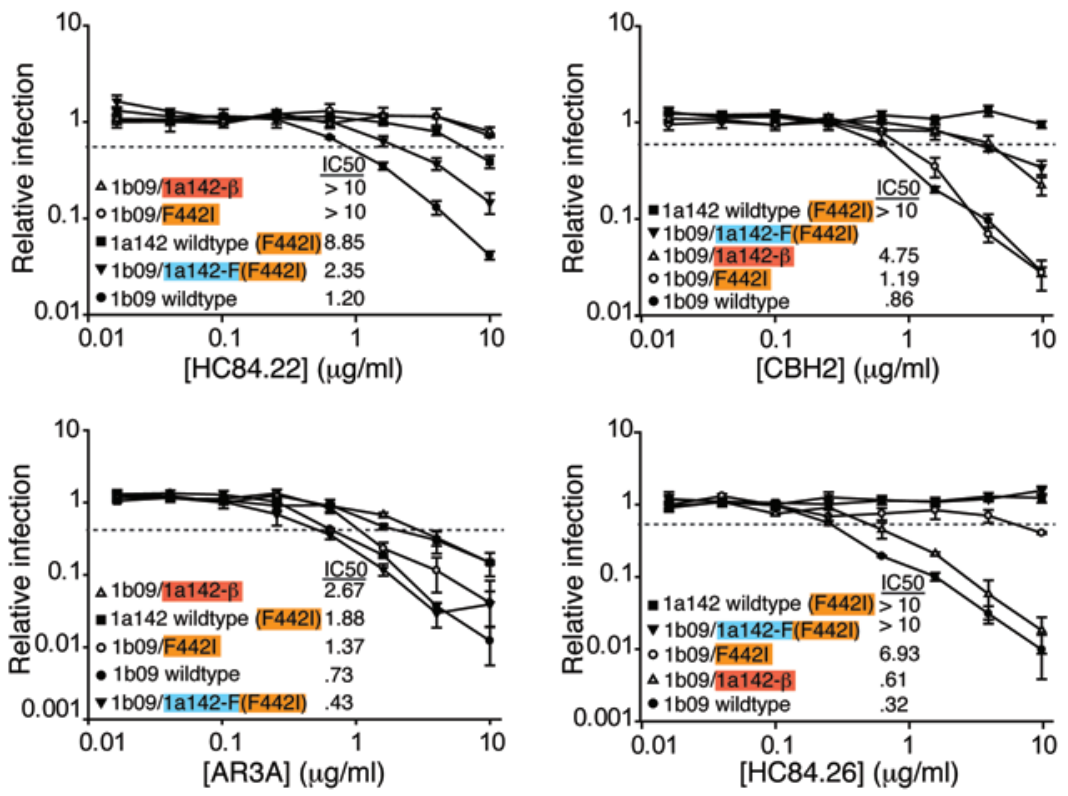

Figure 6. Polymorphisms between amino acids 526 and 569 ( $\beta$-sheet) of resistant E1E2 clone 1 1a142 confer resistance to multiple broadly neutralizing mAbs. Neutralization by the indicated mAb of HCVpp with E1E2 chimeras between resistant clone $1 \mathrm{a} 142$ and sensitive clone 1b09 (1b09/1a142- $\beta$ and 1b09/1a142-F [F442I]), E1E2 with F442I introduced by site-directed mutagenesis (1b09/ F442I), and with resistant E1E2 clone 1a142 (1a142 wild type), which carries F442I naturally. Chimeras with the front layer (1a142-F, amino acids 412-444) from resistant clone 1 a142 are marked with cyan. Chimeras with $\beta 6-\beta 8$ (1a142- $\beta$, amino acids 526-569) from resistant clone 1a142 are marked in red. Orange indicates clones with F442I polymorphisms. Dashed lines indicate 50\% neutralization. Error bars indicate SD between duplicate wells. ically conservative and may have subtle effects on neutralization resistance that are not detectable in vitro using current methods. The effect of some of these minor mutations could be additive, as is the case with I538V/Q546L/T563V. Some of these mutations may also depend on an E1E2 sequence context not tested in this study. Work is ongoing to expand the HCVpp panel and better understand the influence of multiple mutations and E1E2 context on neutralization resistance.

In conclusion, we have developed a novel, rapid method, which uses the natural variation of HCV E1E2 to characterize patterns of resistance to neutralizing mAbs, identifying clusters of mAbs with shared resistance profiles that were not fully predicted by prior epitope mapping. Using this method, we identified naturally selected polymorphisms in E2 at both contact and noncontact residues that confer resistance to multiple broadly neutralizing mAbs and plasma samples. This neutralization clustering analysis suggests that broad resistance polymorphisms at noncontact residues may be a major mechanism of immune evasion by $\mathrm{HCV}$, facilitating viral persistence and presenting a challenge for HCV vaccine development.

\section{Methods}

Source of mAbs. mAbs CBH-4B, CBH-2, CBH-5, CBH-7 (18), HC-1 (19), HC84.22, HC84.26 (12), HC33.4, and HC33.8 (20) were generated in the Foung laboratory. mAbs AR1A, AR2A, AR3A, AR3B, AR3C, AR3D (17), AR4A, AR5A (16) were a gift from Mansun Law (The Scripps Research Institute, La Jolla, California, USA).

Source of plasma. Plasma samples were obtained from the BBAASH (37) cohort (Andrea Cox, Johns Hopkins University School of Medicine). Samples from each of the $18 \mathrm{HCV}$-infected subjects who had previously shown at least $50 \%$ neutralization by $1: 100$ plasma dilution of at least $2 \mathrm{HCVpp}$ in the 19 HCVpp panel were selected.

Production of HCVpp library. Generation and validation of a diverse library of 19 genotype $1 \mathrm{HCVpp}$ and its use for quantitation of neutralizing breadth has been previously described (30). The HCVpp panel used in this study is identical, except that 2 E1E2 clones, $1 \mathrm{~b} 20$ and 1a114, were replaced by related clones $1 \mathrm{~b} 21$ and 1a116, which gave more consistent HCVpp infectivity results. Sequences for E1E2s 1b21 and 1a114 have been deposited in GenBank, accession numbers KM6606289. Plasma samples for production of the library were obtained from HCV-infected subjects in the BBAASH cohort (37), Irish Anti-D cohort (38), and Swan Project (39). An E1E2 alignment consisting of 634 genotype 1 clones previously selected for their representation of the worldwide diversity of genotype 1 sequences was obtained and cleaned using BIOedit (31). The package "seqinr" was used to load data into R (http:// www.r-project.org). An amino acid polymorphism was considered represented by the 19 -clone panel if it was observed in at least $5 \%$ of the worldwide sequences and was observed in the 19-clone panel.

HCVpp production and infectivity screening. HCVpp were produced by lipofectamine-mediated transfection of HCV E1E2 and pNL4-3. Luc.R-E plasmids into HEK293T cells as previously described (40, 41). Neutralization assays were performed as described previously (8). For neutralization at a single $\mathrm{mAb}$ concentration, HCVpp were incubated for 1 hour with $\mathrm{mAb}$ at a final concentration of $10 \mu \mathrm{g} / \mathrm{ml}$ and then added in duplicate to Hep3B target cells for 5 to 6 hours before media were changed. Infection was determined after 3 days by measurement of luciferase activity of cell lysates in RLUs. The majority of HCVpp used in neutralization assays produce RLUs greater than $2 \times 10^{6}$, which is more than 100-fold above background measured using mock pseudoparticles lacking E1E2. All HCVpp used in neutralization assays produced RLUs of at least 200,000, which is at least 10-fold above background. HCVpp with low infectivity were stored at $4^{\circ} \mathrm{C}$ until use to avoid freeze thawing, which reduces infectivity. With a few noted exceptions, HCVpp compared with each other were either all freeze thawed once or all stored at $4^{\circ} \mathrm{C}$ without freeze thawing until use.

Relative infection (neutralization) was calculated by dividing the RLUs produced in the presence of the test mAb ( $\left.\mathrm{RLU}_{\text {test }{ }_{\mathrm{mAb}}}\right)$ at $10 \mu \mathrm{g} /$ $\mathrm{ml}$ by the RLUs of the same HCVpp in the presence of the same concentration of normal human IgG $\left(\mathrm{RLU}_{\text {control IgG }}\right)$. For $\mathrm{IC}_{50}$ determination, HCVpp were incubated with serial 2.5-fold dilutions of mAb, starting from 50 or $10 \mu \mathrm{g} / \mathrm{ml}$, and compared with infection with the same dilutions of normal human IgG. 


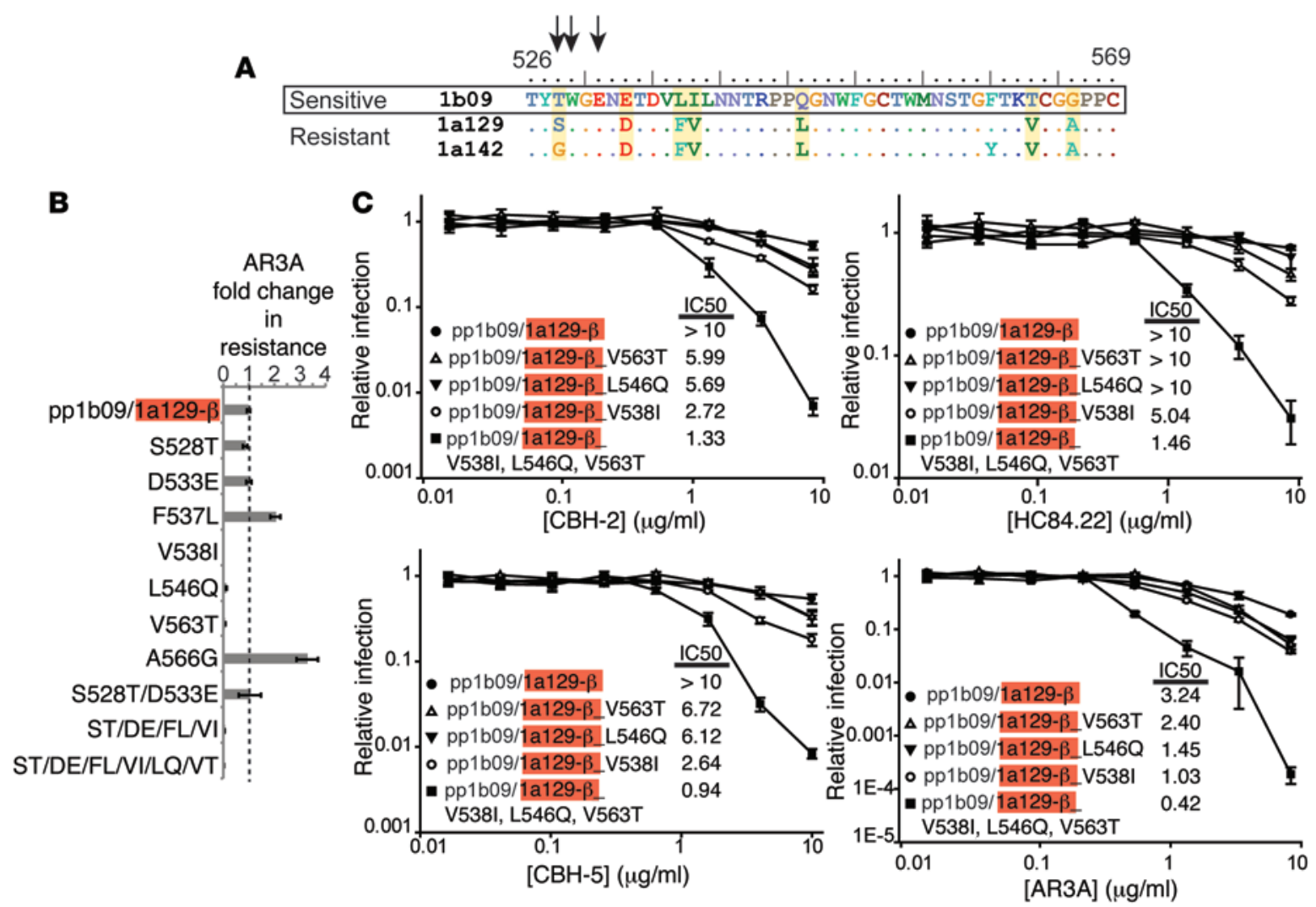

Figure 7. V538I/L546Q/V563T mutations in E2 confer additive sensitivity to NC1 mAbs. (A) Alignment of amino acids 526-569 of sensitive E1E2 clone $1 \mathrm{~b} 09$ and resistant clones $1 \mathrm{a} 129$ and 1a142. Homology to the $1 \mathrm{~b} 09$ amino acid sequence is indicated by a dot. Positions that differ between the sensitive and both resistant clones are highlighted in yellow. Arrows indicate contact residues for AR3C in the E2 core/AR3C crystal structure. (B) The dashed line indicates relative infection of HCVpp with chimeric E1E2 1b09/1a129- $\beta$ in the presence of the mab AR3A, adjusted to 1. Each subsequent bar indicates the fold change in neutralization resistance after the indicated mutation(s) were introduced. Error bars indicate SD between duplicate wells. (C) Neutralization by serial dilutions of the indicated mAb of HCVpp with chimeric E1E2 1b09/1a129- $\beta$ (pp1b09/1a129- $\beta$ ) or pp1b09/1a129- $\beta$ after introduction of the indicated mutation(s). Error bars indicate SD between duplicate wells.

Selection of resistance-associated polymorphisms. E1E2 sequences of neutralization-sensitive clones were compared with those of neutralization-resistant clones using VisSPA v1.6 (http://sray.med.som.jhmi. edu/SCRoftware/VisSPA/) to identify variants present in each resistant E1E2 clone but in none of 5 neutralization-sensitive clones. First, each $\mathrm{E} 1 \mathrm{E} 2$ clone was ranked by increasing resistance to each $\mathrm{mAb}$. The amino acid sequence of each of the $14 \mathrm{E} 1 \mathrm{E} 2$ clones most resistant to each $\mathrm{mAb}$ was compared with the sequences of the $5 \mathrm{E} 1 \mathrm{E} 2$ clones most sensitive to that $\mathrm{mAb}$ to identify polymorphisms present in each resistant clone but absent in all sensitive clones. Sites with polymorphisms in at least 2 resistant clones but in no sensitive clones were then coded according to whether polymorphisms were present more often in the 7 most highly resistant clones or the 7 more moderately resistant clones. These results were included in a summary panel for each mAb. The results for all NC1 mAbs were compiled to identify polymorphic sites that were associated with resistance NC1 neutralizing mAbs.

N430D was tested by site-directed mutagenesis along with other resistance-associated polymorphisms despite appearing in only one resistant clone, since asparagine 430 is a contact residue for $\mathrm{mAb}$ $\mathrm{AR} 3 \mathrm{C}$ in the AR3C/E2 core crystal structure.

Generation of site-directed mutants. Mutations of interest were introduced into E1E2 clones by site-directed mutagenesis using QuikChange Lightning Multi (Stratagene). All clones were sequenced in their entirety after mutagenesis to confirm that no unintended additional mutations were introduced.

Generation of E1E2 chimeras. E1E2 chimeras were generated with E1E2 clone $1 \mathrm{b09}$ and regions encoding amino acids 412-444, amino acids 526-569, and amino acids 412-569 from clones $1 \mathrm{a} 142$ and 1a129. 1b09 E1E2 lacking the nucleotides encoding these regions was amplified by PCR from the cloned 1b09 plasmid using Accuprime Pfx (Invitrogen) and the following primer sets: 5'CD81P11b09, CATAAGTTCAACGCGTCCGGATGCCCAG; 3'CD81Full1b09, GACTCTCTGGGACGCTCCGCTTGAG; 5'CD81Full1b09, AGGGGCGCCGAACCGATCGGTTGTCC; and 3'CD81P21b09, AACATCGGGGGGGTCGGTAACAACACCCTG. The regions encoding amino acids 412-444, 526-569, and 412-569 were amplified by PCR from 1a129 plasmids using the following primers: 5'CD81BS1a129, GCGTCCCAGAGAGTCCAGCTGGTCAACACTAACGGTAGTTGGCAC; 3'CD81BS1a129, GACCCCCCCGATGTTACAGGGGGGCGCTCCGCACACTTTGGTGAATCC; 3'CD81P11a129, CGCGTTGAACTTATGGCGGTAGAAGAGCCCCGCCACCCAGCCG; and 5'CD81P21a129， CGGTTCGGCGCCCCTACCTACAGCTGGGGTGAGAATGATACGGAC. Primers were also modified to match clone 1a142 in order to amplify the same regions from these clones. Primers have 15 base pair $5^{\prime}$ ends with homology to the 1 b09 sequence. Chimeras were then constructed using In-Fusion cloning (Clontech). All chi- 

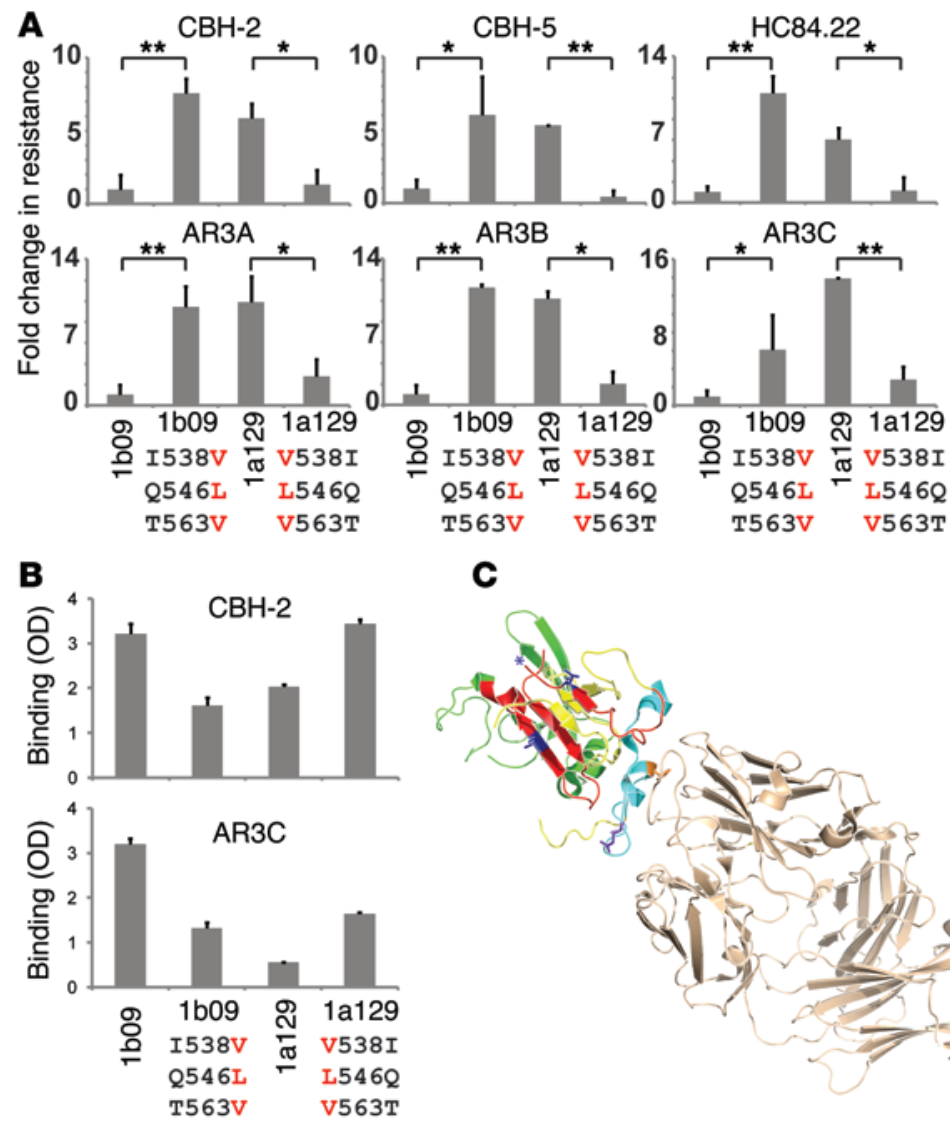

C

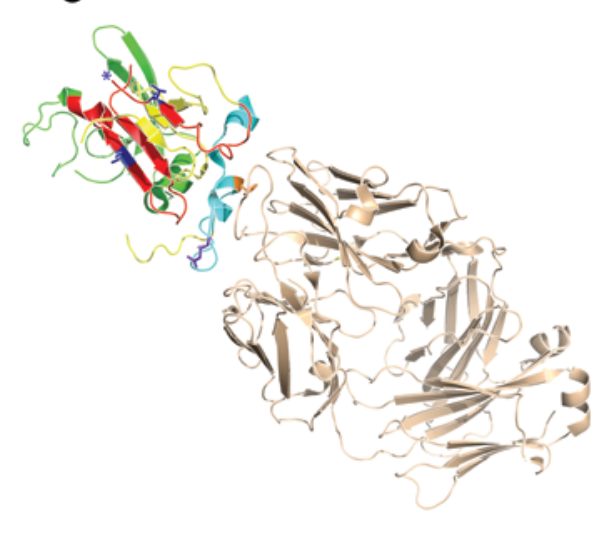

Figure 8. I538V/Q546L/T563V mutations in E2 confer resistance to 6 NC1 mAbs by reducing $\mathrm{mAb}$ binding to E1E2. (A) The first bar in each graph indicates relative infection of wild-type pp1b09 in the presence of the indicated mAb, adjusted to 1 . Subsequent bars indicate fold change in neutralization resistance of the indicated HCVpp relative to pp1b09. Values are the means of 2 to 6 independent experiments performed in duplicate, and error bars indicate SD. ${ }^{*} P<0.05,{ }^{* *} P<0.005$ by $t$ test. (B) Binding of mAbs CBH-2 and AR3C to E1E2 protein in an ELISA. For each mAb, binding to each of the E1E2 variants was compared at a single mAb concentration selected to produce binding within the linear range of the assay. Values are normalized for relative binding of control NC2 mAb HC33.4. Error bars indicate SD between duplicate wells. (C) Crystal structure from Kong et al. of E2 core with AR3C (34), from the Protein Data Bank, accession 4MWF, with E2 colors modified as in Figure 5. Residue 431 is purple, residue 442 is orange, and residues 538 and 563 are blue. The likely position of residue 546 is indicated with a blue asterisk. AR3C Fab is tan.

HCV NS5A immunostaining. Huh7.5.1 cells plated at 10,000 cells per well on a 96-well plate the previous day were incubated with viral supernatants overnight. 72 hours after infection, cells were fixed with $4 \%$ formaldehyde for 20 minutes and then stained for HCV NS5A using primary anti-NS5A antibody 9E10 (a gift of Charles Rice, The Rockefeller University, New York City, New York, USA) at 1:20,000 dilution in PBS, $3 \%$ bovine serum albumin, and $0.3 \%$ Triton $\mathrm{X}-100$ for 1 hour at room temperature. Cells were washed twice with PBS and stained using secondary antibody Alexa Daylight 488-conjugated goat anti-mouse IgG (Life Technologies) at 1:1,000 dilution in PBS, 3\% meric E1E2 clones were sequenced in their entirety after construction to confirm that no unintended mutations were introduced.

Production and neutralization of HCVcc. Human hepatoma Huh7.5.1 cells (a gift of Jake Liang, NIH, Bethesda, Maryland, USA) were maintained in DMEM supplemented with $10 \%$ fetal bovine serum, $1 \%$ sodium pyruvate, and $1 \%$ L-glutamate. HCVcc chimera H77/JFH1 (32) was a gift of Jens Bukh (Copenhagen University Hospital, Copenhagen, Denmark). $30 \mu$ g plasmid DNA was linearized using XBAI (New England Biolabs) and then gel purified using the QIAEX II Kit (Qiagen). Purified linear DNA was used for in vitro RNA transcription using the T7 RiboMAX Express Large Scale RNA Production System (Promega). A phenol-chloroform extraction was performed to purify RNA products that were then stored at $-80^{\circ} \mathrm{C}$.

Transfection of Huh 7.5.1 cells with HCVcc RNA was performed as previously described (29). Transfection supernatants were collected every 3 to 4 days and stored at $-80^{\circ} \mathrm{C}$ for titering by $\mathrm{HCV}$ NS5A immunostaining.

Figure 9. $1538 \mathrm{~V} / \mathrm{Q} 546 \mathrm{~L} / \mathrm{T} 563 \mathrm{~V}$ mutations in E2 confer resistance to neutralization by plasma from HCV-infected subjects. Introduction of I538V/ Q546L/T563V into sensitive E1E2 clone 1b09 or back mutation of V538 to I, L546 to Q, and V563 to T in resistant clone 1 a129 confers increased resistance or sensitivity, respectively, to neutralization by plasma samples from $18 \mathrm{HCV}$-infected subjects. Each box represents relative infection of the indicated HCVpp in the presence of each of 18 plasma samples. Dots indicate neutralization by plasma from the source donor of resistant E1E2 clone 1a129. Plasma was tested for neutralizing activity at a 1:100 dilution. Black bars indicate medians. Whiskers indicate 10th and 90th percentiles. $P$ values were calculated by Wilcoxon signed-rank test. bovine serum albumin, and $0.3 \%$ Triton $\mathrm{X}-100$ for 1 hour at room temperature. Cells were washed twice in PBS and then stored covered in $100 \mu \mathrm{l} \mathrm{PBS}$ at $4^{\circ} \mathrm{C}$. To determine peak infectivity, wells were examined with a Zeiss invert microscope with an Olympus camera. Viral supernatants with peak infectivity were kept for further experiments.

HCVcc neutralization assays. Huh7.5.1 cells were plated at 10,000 cells per well onto 96-well plates. On the following day, antibodies were serially diluted 2.5-fold in growth medium, starting from $50 \mu \mathrm{g} / \mathrm{ml} \mathrm{mAb}$. Three to six replicateswere also performed with $50 \mu \mathrm{g} / \mathrm{ml}$ nonspecific IgG.

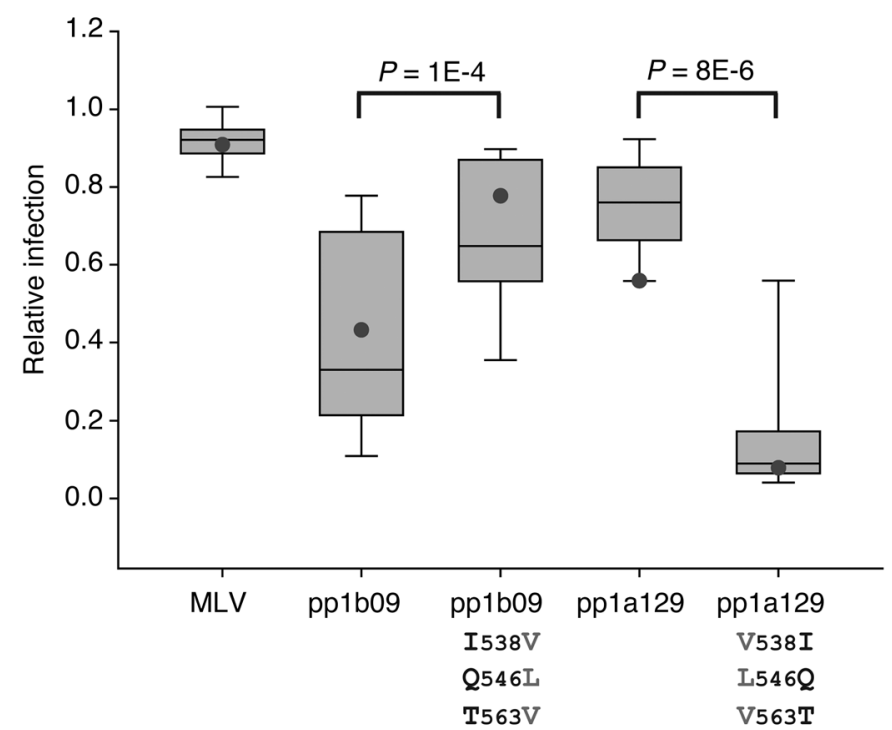


Viral supernatants diluted to fall in a linear range of infectivity were added to antibody dilutions in triplicate for 1 hour at $37^{\circ} \mathrm{C}$. Medium was removed from cells, and the antibody-virus mix added onto cells and incubated overnight. Antibody-virus mix was replaced with fresh medium the following day. After 72 hours, medium was removed and cells were fixed and stained. A composite image of each well was created using an LSM 510 Meta confocal microscope. Images were analyzed using Image J to quantify the total area of fluorescence in each well. Neutralization was calculated by calculating the ratio of infection at each concentration of $\mathrm{mAb}$ to infection in the presence of nonspecific IgG or the most dilute $\mathrm{mAb}$ concentration, whichever had a higher mean value.

HCV E1E2 ELISA. mAb binding to E1E2 was quantitated using an ELISA as previously described (14). Briefly, 293T cells were transfected with one of the following E1E2 expression constructs: wild-type 1b09, 1b09 with I538V/Q546L/T563V introduced by site-directed mutagenesis, wild-type 1a129, or 1a129 with V538I/ L546Q/V563T introduced by site-directed mutagenesis. Cell lysates were harvested at 48 hours. Plates were coated with 500 ng Galanthus nivalis lectin (Sigma-Aldrich) and blocked with PBS containing $0.5 \%$ Tween $20,1 \%$ nonfat dry milk, and $1 \%$ goat serum, and E1E2containing cell lysates were added. mAbs were assayed in duplicate at 5 -fold serial dilutions, starting at $10 \mu \mathrm{g} / \mathrm{ml}$, and binding was detected with HRP-conjugated anti-human IgG secondary antibody (BD Pharmingen no. 555788). For each mAb, a dilution was chosen that produced binding within the linear range of the assay (OD 0.13.5) for all variants, and binding to each E1E2 variant was compared at that $\mathrm{mAb}$ concentration. Binding of each $\mathrm{mAb}$ was normalized for protein expression by correcting for any difference in binding of control NC2 mAb HC33.4 between E1E2 variants.

Hierarchical clustering of $n A b$ patterns. Effects on infectivity of the panel of HCVpp by mAbs were compared pairwise for all mAbs using the Spearman correlation. Spearman rho and $P$ values were then used as input for hierarchical clustering, as implemented in the "pvclust" package for R (http://cran.r-project.org/web/packages/pvclust/index.html)
(42). Approximately unbiased values greater than 95 are considered strongly supported by the data. This clustering, depicted as a tree, was also used to order a matrix of correlation values produced using the "corrplot” package for R (http://cran.r-project.org/web/packages/corrplot/ index.html) (43).

Statistics. Neutralization curves for HCVpp and HCVcc were fit by nonlinear regression in SigmaPlot, and $\mathrm{mAb} \mathrm{IC}_{50}$ was calculated based on that curve. Significance of the difference in proportion of positions with polymorphisms between amino acids 416 and 560 relative to the rest of the E1E2 sequence (Figure 3) was calculated using a $\mathrm{z}$ test. Comparison of relative infection (neutralization) of wild-type clones and site-directed mutants by mAbs (Figures 4 and 8 ) was performed using 1-sided $t$ tests, since prior evidence supported increased resistance of mutant clones. Comparison of relative infection (neutralization) of wild-type clones and site-directed mutants by donor plasma (Figure 9) was performed using Wilcoxon signed-rank test. Comparison of infectivity (fitness) of wild-type and mutant clones (Supplemental Figure 10) was performed using 1-sided $t$ tests. In all cases, a $P$ value of less than 0.05 was considered significant.

Study approval. All protocols were approved by the Institutional Review Board of the Johns Hopkins Hospital, and informed consent was obtained from all study participants.

\section{Acknowledgments}

We would like to thank David L. Thomas for helpful discussions and Joel N. Blankson for helpful discussions and thoughtful reading of this manuscript. This study was supported by NIH grants 1K08 AI102761, U19 AI088791, and R37 DA013806 and the Johns Hopkins University Center for AIDS Research grant 1P30AI094189.

Address correspondence to: Stuart C. Ray, Center for Viral Hepatitis Research, Johns Hopkins University, 855 N. Wolfe Street, Suite 530, Baltimore, Maryland 21205, USA. Phone: 410.614.2891; E-mail: sray@jhmi.edu.
1. Lawitz E, et al. Sofosbuvir in combination with peginterferon alfa-2a and ribavirin for non-cirrhotic, treatment-naive patients with genotypes 1, 2, and 3 hepatitis $\mathrm{C}$ infection: a randomised, double-blind, phase 2 trial. Lancet Infect Dis. 2013;13(5):401-408.

2. Cox AL, Thomas DL. Hepatitis $C$ virus vaccines among people who inject drugs. Clin Infect Dis. 2013;57(suppl 2):S46-S50.

3. Liang TJ. Current progress in development of hepatitis $\mathrm{C}$ virus vaccines. Nat Med. 2013;19(7):869-878.

4. Dienstag JL. The natural history of chronic hepatitis $C$ and what we should do about it. Gastroenterology. 1997;112(2):651-655.

5. Liu L, Fisher BE, Dowd KA, Astemborski J, Cox $\mathrm{AL}$, Ray SC. Acceleration of hepatitis $\mathrm{C}$ virus envelope evolution in humans is consistent with progressive humoral immune selection during the transition from acute to chronic infection. J Virol. 2010;84(10):5067-5077.

6. Liu L, Fisher BE, Thomas DL, Cox AL, Ray SC. Spontaneous clearance of primary acute hepatitis $C$ virus infection correlated with high initial viral RNA level and rapid HVR1 evolution. Hepatology.
2012;55(6):1684-1691.

7. Simmonds P. Genetic diversity and evolution of hepatitis C virus - 15 years on. J Gen Virol. 2004;85(pt 11):3173-3188.

8. Dowd KA, Netski DM, Wang XH, Cox AL, Ray SC. Selection pressure from neutralizing antibodies drives sequence evolution during acute infection with hepatitis C virus. Gastroenterology. 2009;136(7):2377-2386.

9. Farci $\mathrm{P}$, et al. Prevention of hepatitis $\mathrm{C}$ virus infection in chimpanzees after antibody-mediated in vitro neutralization. Proc Natl Acad Sci US A. 1994;91(16):7792-7796.

10. Shimizu YK, Hijikata M, Iwamoto A, Alter HJ, Purcell RH, Yoshikura H. Neutralizing antibodies against hepatitis $\mathrm{C}$ virus and the emergence of neutralization escape mutant viruses. J Virol. 1994;68(3):1494-1500.

11. von Hahn T, et al. Hepatitis $C$ virus continuously escapes from neutralizing antibody and T-cell responses during chronic infection in vivo. Gastroenterology. 2007;132(2):667-678.

12. Keck ZY, et al. Human monoclonal antibodies to a novel cluster of conformational epitopes on $\mathrm{HCV}$ e2 with resistance to neutralization escape in a genotype 2a isolate. PLoS Pathog. 2012;8(4):e1002653.

13. Keck ZY, et al. Mapping a region of hepatitis $\mathrm{C}$ virus $\mathrm{E} 2$ that is responsible for escape from neutralizing antibodies and a core CD81-binding region that does not tolerate neutralization escape mutations. J Virol. 2011;85(20):10451-10463.

14. Keck ZY, et al. Mutations in hepatitis $C$ virus E2 located outside the CD81 binding sites lead to escape from broadly neutralizing antibodies but compromise virus infectivity. J Virol. 2009;83(12):6149-6160.

15. Kong L, et al. Structural basis of hepatitis C virus neutralization by broadly neutralizing antibody HCV1. Proc Natl Acad Sci U S A. 2012;109(24):9499-9504.

16. Giang E, et al. Human broadly neutralizing antibodies to the envelope glycoprotein complex of hepatitis C virus. Proc Natl Acad Sci U S A. 2012;109(16):6205-6210.

17. Law $\mathrm{M}$, et al. Broadly neutralizing antibodies protect against hepatitis $\mathrm{C}$ virus quasispecies challenge. Nat Med. 2008;14(1):25-27.

18. Hadlock KG, et al. Human monoclonal antibodies that inhibit binding of hepatitis $\mathrm{C}$ virus $\mathrm{E} 2$ protein 
to CD81 and recognize conserved conformational epitopes. J Virol. 2000;74(22):10407-10416.

19. Keck ZY, et al. A point mutation leading to hepatitis $C$ virus escape from neutralization by a monoclonal antibody to a conserved conformational epitope. JVirol. 2008;82(12):6067-6072.

20. Keck Z, et al. Cooperativity in virus neutralization by human monoclonal antibodies to two adjacent regions located at the amino terminus of hepatitis C virus E2 glycoprotein. J Virol. 2013;87(1):37-51.

21. Krey T, Meola A, Keck ZY, Damier-Piolle L, Foung SK, Rey FA. Structural basis of HCV neutralization by human monoclonal antibodies resistant to viral neutralization escape. PLoS Pathog. 2013;9(5):e1003364.

22. Bartosch B, et al. In vitro assay for neutralizing antibody to hepatitis $C$ virus: evidence for broadly conserved neutralization epitopes. Proc Natl Acad Sci US A. 2003;100(24):14199-14204.

23. Broering TJ, et al. Szabo G, Finberg RW, Ambrosino DM, and Babcock GJ. Identification and characterization of broadly neutralizing human monoclonal antibodies directed against the E2 envelope glycoprotein of hepatitis C virus. JVirol. 2009;83(23):12473-12482.

24. Johansson DX, et al. Human combinatorial libraries yield rare antibodies that broadly neutralize hepatitis C virus. Proc Natl Acad Sci U S A. 2007;104(41):16269-16274.

25. Perotti M, et al. Identification of a broadly cross-reacting and neutralizing human monoclonal antibody directed against the hepatitis $C$ virus E2 protein. J Virol. 2008;82(2):1047-1052.

26. Gottwein JM, et al. Development and charac- terization of hepatitis $C$ virus genotype 1-7 cell culture systems: role of CD81 and scavenger receptor class B type I and effect of antiviral drugs. Hepatology. 2009;49(2):364-377.

27. Tarr AW, et al. Hepatitis $C$ patient-derived glycoproteins exhibit marked differences in susceptibility to serum neutralizing antibodies: genetic subtype defines antigenic but not neutralization serotype. JVirol. 2011;85(9):4246-4257.

28. Bartosch B, Dubuisson J, Cosset FL. Infectious hepatitis $\mathrm{C}$ virus pseudo-particles containing functional E1- E2 envelope protein complexes. JExp Med.2003;197(5):633-642.

29. Wakita T, et al. Production of infectious hepatitis $\mathrm{C}$ virus in tissue culture from a cloned viral genome. Nat Med. 2005;11(7):791-796.

30. Osburn WO, et al. Clearance of hepatitis C infection is associated with the early appearance of broad neutralizing antibody responses. Hepatology. 2014;59(6):2140-2151

31. Munshaw S, et al. Computational reconstruction of bole1a, a representative synthetic hepatitis C virus subtype 1a genome. J Virol. 2012;86(10):5915-5921.

32. Scheel TK, et al. Development of JFH1-based cell culture systems for hepatitis $C$ virus genotype $4 \mathrm{a}$ and evidence for cross-genotype neutralization. Proc Natl Acad Sci U S A. 2008;105(3):997-1002.

33. Law M, et al. Broadly neutralizing antibodies protect against hepatitis $\mathrm{C}$ virus quasispecies challenge. Nat Med. 2008;14(1):25-27.

34. Kong L, et al. Hepatitis C virus E2 envelope glycoprotein core structure. Science. 2013;342(6162):1090-1094.
35. Carlsen TH, et al. Breadth of neutralization synergy of clinically relevant human monoclonal antibodies against $\mathrm{HCV}$ genotypes 1a, 1b, 2a, 2b, 2c, 3a. Hepatology. 2014;60(5):1551-1562.

36. Fofana I, et al. Mutations that alter use of hepatitis $C$ virus cell entry factors mediate escape from neutralizing antibodies. Gastroenterology. 2012;143(1):223-233.

37. Cox AL, et al. Prospective evaluation of community-acquired acute-phase hepatitis $\mathrm{C}$ virus infection. Clin Infect Dis. 2005;40(7):951-958.

38. Kenny-Walsh E. Clinical outcomes after hepatitis Cinfection from contaminated anti-D immune globulin. Irish Hepatology Research Group. N Engl JMed.1999;340(16):1228-1233.

39. Raghuraman S, Park H, Osburn WO, Winkelstein E, Edlin BR, Rehermann B. Spontaneous clearance of chronic hepatitis $C$ virus infection is associated with appearance of neutralizing antibodies and reversal of T-cell exhaustion. J Infect Dis. 2012;205(5):763-771.

40. Hsu M, et al. Hepatitis C virus glycoproteins mediate $\mathrm{pH}$-dependent cell entry of pseudotyped retroviral particles. Proc Natl Acad Sci U S A. 2003;100(12):7271-7276.

41. Logvinoff C, et al. Neutralizing antibody response during acute and chronic hepatitis C virus infection. Proc Natl Acad Sci U S A. 2004;101(27):10149-10154.

42. Suzuki R, Shimodaira H. Pvclust: an R package for assessing the uncertainty in hierarchical clustering. Bioinformatics. 2006;22(12):1540-1542.

43. Friendly M. Corrgrams: Exploratory displays for correlation matrices. Am Stat. 2002;56(4):316-324. 